\title{
EDITORIAL
}

\section{New approaches to the treatment of pulmonary fibrosis}

\author{
R.A. DeRemee
}

These observations come from a pulmonologist who has studied and treated diffuse interstitial pulmonary disease for almost a third of a century in a large academic referral centre [1]. The most vexing of the entities encountered has been pulmonary fibrosis. The latter condition and sarcoidosis are the two most common interstitial pulmonary diseases seen at Mayo Clinic. Since the 1950s, glucocorticoids have been used in the treatment of pulmonary fibrosis, either alone or in combination with other agents. In more recent times, cytotoxic drugs, such as cyclophosphamide or azathioprine, have been used with or without a glucocorticoid. In the past 20 years, a number of studies have appeared in the literature concerning the effects of these regimens [2-8]. The results have been uniformally disappointing, with only marginal improvement in survival. In fact, the data reported, and this author's personal experience, demand that we question whether it is any longer worthwhile to perform and report such studies with these agents? When will we be convinced that they do not cure, have only minimal positive impact on the patient, and often incur intolerable side-effects? Pulmonary fibrosis tends to occur in older patients, who are particularly vulnerable to the side-effects of glucocorticoids, especially women due to their propensity for osteoporosis.

One area needing new consideration is that of definitions. Are we all talking about the same disease, variously called idiopathic pulmonary fibrosis (IPF), or cryptogenic fibrosing alveolitis (CFA)? In this author's view, many investigators have not set down firm clinicopathological criteria, and it seems that any diffuse fibrosis of the lung that has no apparent cause can be considered under the heading of IPF. Others suggest that any of the major diffuse entities, such as sarcoidosis, histiocytosis X, hypersensitivity pneumonitis, etc., can evolve into a clinical picture indistinguishable from IPF. Still others are loath to accept any case as IPF if it has features of the so-called collagen vascular or connective tissue diseases, such as rheumatoid arthritis, systemic lupus erythematosus, or scleroderma. The validity of any study is influenced by the homogeneity of the patients studied, which implies specific criteria for diagnosis. In 1972, because of the ambiguity in the literature in this respect, we developed the concept of classic interstitial pneumonitis-fibrosis or CIP-F, wherein specific criteria were prescribed [9]. The concept avoids the term "idiopathic" and, therefore, includes

Mayo Medical School, Rochester, MN 55905, USA. many instances of overlap with various collagen diseases. We must remember that terms such as systemic lupus erythematosus, rheumatoid arthritis, scleroderma, and the like are not immutable, but only artificial mental models of common manifestations, allowing physicians to deal with these problems. However, our mental models can be altered by accumulation of new data or new conceptual insights. Certainly, all the entities above are idiopathic. The fact that a patient has pulmonary fibrosis and may have overlap features of collagen vascular disease, does not make the pulmonary fibrosis component "un-idiopathic". It is quite possible that our perpetuation of the term idiopathic in this area of discussion may subtly divert our creative thinking, by disallowing the common features seen in pulmonary fibrosis and the collagen disease spectrum.

No doubt a major road to treatment will be the systematic dissection of the intricate cascade of cellular and humoral events leading to fibrosis. The articles by PIGUET and Vesin [10], concerning the modification of pulmonary fibrosis in experimental mice by the administration of an antagonist to tumour necrosis factor, provides the kind of information yielding insight into new treatment. The same can be said for the study reported by Meyer et al. [11] about the use of oral Nacetylcysteine in human subjects to counter the oxidant imbalance in the lung of patients with fibrosis. The obvious reservations and cautions concerning any new treatments relate to their tolerance, long- and short-term side-effect, and cost. We must also be cautious about an inordinate focus on the end result of fibrosis, to the exclusion of the events which precede it. For example, consider a destroyed city. Clues to its destruction or derangement may not be readily ascertained by simply searching about in the ruins. Was it caused by a bomb, an artillery shell, an artificial intrinsic explosion, a storm? All these forces may result in identical final results. What is most important in preventing similar events is the identification and interdiction of the vehicle, mode of injury, or the specific aetiological agent or agents. That is to say, should we guard against the bomber, the artillery piece, or whatever? In this sense, an awareness of various and diverse entities that lead to a fibrotic conclusion is essential. For instance, how do the mechanisms involved in sarcoidosis relate to those of histiocytosis X, CIP-F, or other diffuse interstitial diseases? As suggested previously, might not CIP-F be considered a part of the collagen disease spectrum, with the implication that similar pathogenic 
mechanisms are operative? Given the marvellous new tools of cellular and molecular biology, the future seems reasonably bright in elucidating these mechanisms.

But what should we do today for our patients with fibrosis? It has already been suggested that traditional regimens with glucocorticoids and/or cytotoxic drugs are, at best, of marginal impact. In many cases, the side-effects of these regimens outweigh the benefits. Given the dismal results of traditional regimens and the encouraging, albeit anecdotal, results with colchicine, the Mayo group has embarked on a prospective study, comparing colchicine with prednisone in a randomized cohort [12]. Should colchicine prove as effective, or indeed more effective, than prednisone, we will have found a much less toxic replacement for glucocorticoids. A prime consideration in any treatment strategy must be early detection. Quite probably, once alveoli have been eliminated or distorted by the fibrotic process, there is little hope of restoring normal architecture. Lung transplantation is currently a logical, but hardly practical, alternative for the large number of patients needing relief from their distressing symptoms. At our institution, the average estimated cost of a lung transplantation is $\$ 100,000$. Moreover, only about one half of patients who apply qualify for the procedure, and the number of available organs is much too small to satisfy the need.

While some labour at the scientific bench, clinicians should not be afraid of conducting treatment studies based on reasonable empiricism. Reasonable empiricism can mean the use of drugs, of known action and safety, in new situations, based on previously observed positive effects in similar diseases or the theoretical possible efficacy. Such is the case underlying the study with colchicine. Other agents, such as those used in other inflammatory diseases, should be considered, e.g. antimalarials and antiprostaglandins.

Physicians must guard against overtreatment in dire situations. It is not good medicine to apply toxic, unproven regimens to patients with progressive, incurable disease simply to give the appearance of action. In many cases, such treatments are given to assuage the physician's feelings of therapeutic inadequacy. Many patients are better served by simply giving reasonable, nonspecific support, be it mental, spiritual or medical. As many of our patients with fibrosis will be hypoxic, it is of more than passing interest to question the role of supplemental oxygen for a disease theoretically due to oxidant injury. Whilst we study and await scientific breakthroughs, we must not forget a guiding concept of medicine, primum non nocere.

\section{References}

1. DeRemee RA. Clinical profiles of diffuse interstitial pulmonary disease. New York, Futura Publishing Co., 1990.

2. Turner-Warwick M, Burrows B, Johnson A. Cryptogenic fibrosing alveolitis: clinical features and their influence on survival. Thorax 1980; 35: 171-180.

3. Rudd RM, Haslam PL, Turner-Warwick M. Cryptogenic fibrosing alveolitis: relationships of pulmonary physiology and bronchoalveolar lavage to response to treatment and prognosis. Am Rev Respir Dis 1981; 124: 1-8.

4. Turner-Warwick M, Burrows B, Johnson A. Cryptogenic fibrosing alveolitis: response to corticosteroid treatment and its effect on survival. Thorax 1980; 35: 593-599.

5. Winterbauer RH, Hammar SP, Hallman KO, et al. Diffuse interstitial pneumonitis: clinicopathologic correlations in 20 patients treated with prednisone/azathioprine. Am J Med 1978; 65: 661-672.

6. Raghu G, Depaso WJ, Cain K, et al. Azathioprine combined with prednisone in the treatment of idiopathic pulmonary fibrosis: a prospective double-blind, randomized, placebo-controlled trial. Am Rev Respir Dis 1991; 144: 291-296.

7. Johnson MA, Kwan S, Snell NJ, Nunn AJ, Darbyshire JH, Turner-Warwick M. Randomized controlled trial comparing prednisolone alone with cyclophosphamide and low dose prednisolone in combination in cryptogenic fibrosing alveolitis. Thorax 1989; 44; 280-288.

8. Meier-Sydow J, Rust M, Kronenberger H, Thiel C, Amthor M, Riemann H. Long-term follow-up of lung function parameters in patients with idiopathic pulmonary fibrosis treated with prednisone and azathioprine or D-penicillamine. Prax Pneumol 1979; 33: 680-688.

9. DeRemee RA, Harrison EG, Andersen HA. The concept of classic interstitial pneumonitis-fibrosis (CIPF) as a clinicopathologic syndrome. Chest 1972; 61: 213-220.

10. Piguet PF, Vesin C. Treatment by human recombinant soluble TNF receptor of pulmonary fibrosis induced by bleomycin or silica in mice. Eur Respir $J$ 1994; 7 : 515-518.

11. Meyer A, Buhl R, Magnussen H. The effect of oral Nacetylcysteine on lung glutathione levels in idiopathic pulmonary fibrosis. Eur Respir J 1994; 7: 431-436.

12. Peters SG, McDougall JC, Douglas WW, Coles DT, DeRemee RA. Colchicine in the treatment of pulmonary fibrosis. Chest 1993; 103: 101-104. 